\title{
TWO APPLICATIONS OF THE TECHNIQUE OF LENGTH-DECREASING VARIATIONS
}

\author{
NATHANIEL GROSSMAN
}

By the "technique of length-decreasing variations," we mean a method used by Synge, Morse, Frankel, and many others. The method consists of showing that certain minimization problems in the theory of geodesics in the large, frequently associated with topological questions, cannot have a nontrivial solution by proving that any extremal for the problem has a variation in to nearby curves of strictly smaller length. Thus, Synge [8] shows that an even-dimensional orientable compact manifold of positive sectional curvatures must be simply-connected. Frankel [2], [3] shows that two compact minimal hypersurfaces in a manifold of positive Ricci curvatures must intersect. Morse [6] studies e.g. critical chords of submanifolds of Euclidean space.

In Part I, we study hypersurfaces in manifolds of positive Ricci curvatures. Frankel [3] has shown that the fundamental groups of such manifolds are homomorphs of the fundamental groups of their minimal hypersurfaces. We reprove this theorem by a slightly different method from that used by Frankel and generalize it by allowing the hypersurfaces to be not necessarily minimal. If suitable curvature hypotheses are satisfied, the fundamental group of the manifold is a homomorph of the fundamental group of a certain component of the complement of the hypersurface. A precise statement of the result is given as Theorem 1 .

Klingenberg has proved that under various hypotheses on the sectional curvatures, connectivity, and dimension of a manifold, its injectivity radii are $\geqq \pi$ (cf. [5]). In Part II, we introduce the "thickness" of an embedded hypersurface, dual to the injectivity radius of a point, and show in Theorem 2 that, in a manifold of positive sectional curvatures bounded above by 1 , the thickness of a separating totally geodesic hypersurface is $\geqq \pi / 2$. In case a separating hypersurface is not totally geodesic, we show in Theorem 3 that, in one of the components of its complement, it may have a collar neighborhood whose thickness can be explicitly estimated.

All manifolds will be smooth with a complete Riemannian metric. $M$ will be a manifold of dimension $n$ with a smoothly embedded hypersurface $N$. Let the metric on $M$ be denoted by $\langle\cdot, \cdot\rangle$ and the asso-

Received by the editors March 28, 1966. 
ciated norm by $\|\cdot\|$. Let $N^{\perp}$ be the normal bundle of the embedding $N \subset M$ with induced norm on the fibers. Let $\Re^{\perp} \subset N^{\perp}$ be the subset of $v \in N^{\perp}$ with $\|v\|=1$. Let exp be the exponential map of the LeviCivita connection $\nabla$ of $\langle\cdot, \cdot\rangle$ and denote its restriction to $N^{\perp}$ again by exp.

I. Call $c:[a, b] \rightarrow M$ an $N$-curve if $\dot{c}(a), \dot{c}(b) \in N^{\perp}$ and an $N$-geodesic if it is a geodesic parametrized by arc-length. A variation $V:[a, b] \times J$ $\rightarrow M$ ( $J$ an open interval around 0$)$ will be called an $N$-variation if each $V_{\epsilon}$ given by $V_{\epsilon}(t)=V(t, \epsilon)$ is an $N$-curve.

Let $p \in N, \nu \in\left(\Re^{\perp}\right)_{p}$. The second fundamental form of $N$ at $\nu$ is given by

$$
S_{\nu}(X, Y)=-\left\langle\nabla_{X} \nu, Y\right\rangle
$$

for $X, Y \in N_{p}$. Let $L(\epsilon)=\mathfrak{L}\left(V_{\epsilon}\right)$ be the length of $V_{\epsilon}$. The index form for $\sigma$ is given by

$$
\begin{aligned}
I_{\sigma}(X)= & -S_{\dot{\sigma}(b)}(X(b), X(b))+S_{\dot{\sigma}(a)}(X(a), X(a)) \\
& +\int_{a}^{b}\left\{\left\|\nabla_{t} X(t)\right\|^{2}-K(X(t))\|X(t)\|^{2}\right\} d t
\end{aligned}
$$

where $\nabla_{t}$ is covariant differentiation along $\sigma, X$ is a vector field along $\sigma$ and normal to it, and $K(X(t))$ is the sectional curvature of the twoplane spanned by $X(t)$ and $\dot{\sigma}(t)$ (and $=0$ if $X(t)=0$ ). If $V:[a, b] \times J$ $\rightarrow M$ is an $N$-variation of the $N$-geodesic $\sigma$, then $L^{\prime \prime}(0)=I_{\sigma}(X)$, where $X(t)=\partial V_{\epsilon}(t, 0) / \partial \epsilon$.

As a matter of notation, let $\mathcal{F}_{\sigma}$ be the set of vector fields along $\sigma$, normal to it, autoparallel, and unitary.

If $X \in \mathcal{F}_{\sigma}$, (1) becomes

(2) $I_{\sigma}(X)=-S_{\dot{\sigma}(b)}(X(b), X(b))+S_{\dot{\sigma}(a)}(X(a), X(a))-\int_{a}^{b} K(X(t)) d t$.

If $X_{1}, \cdots, X_{n-1} \in \mathcal{F}_{\sigma}$ are mutually orthogonal, we obtain from (2):

$$
\begin{aligned}
\sum_{m=1}^{n-1} I_{\sigma}\left(X_{n}\right)=-\sum_{m=1}^{n-1} & S_{\dot{\sigma}(b)}\left(X_{m}(b), X_{m}(b)\right) \\
& +\sum_{m=1}^{n-1} S_{\dot{\sigma}(a)}\left(X_{m}(a), X_{m}(a)\right)-(n-1) \int_{a}^{b} R(t) d t,
\end{aligned}
$$

where $R(t)=(n-1)^{-1} \sum_{m=1}^{n-1} K\left(X_{m}(t)\right)$ is the Ricci curvature of $M$ at $\dot{\sigma}(t)$. We note also that the first sum on the right side of $(3)$ is the mean curvature of $N$ at $\dot{\sigma}(b)$, with a similar interpretation for the second sum. 
Suppose the hypersurface $N$ separates $M$. The unit normal bundle $\Re^{\perp}$ separates into two components, the orientations of $N$, and these components correspond in an obvious way to the components of $M-N$. Say that $N$ has semidefinite mean curvatures if the mean curvatures are nonnegative on one orientation of $N$ and nonpositive on the other. Call a component $U$ of $M-N$ exterior to $N$ if the mean curvatures of $N$ are nonnegative on the orientation of $N$ corresponding to $U$.

Theorem 1. Let $M$ have Ricci curvatures $>0$. Let the compact connected hypersurface $N$ separate $M$ and have semidefinite mean curvatures. If $U$ is the component of $M-N$ exterior to $N$, then the natural homomorphism $\pi_{1}(U) \rightarrow \pi_{1}(M)$ is surjective.

Proof. By the exactness of the sequence $\pi_{1}(U) \rightarrow \pi_{1}(M) \rightarrow \pi_{1}(M, U)$, it is enough to show that $\pi_{1}(M, U)=\pi_{1}(M, \bar{U})$ is trivial.

First of all, suppose $\sigma:[a, b] \rightarrow M$ to be a minimal $N$-geodesic such that $\dot{\sigma}(a)$ points "out of $U$ " and $\dot{\sigma}(b)$ points "into $U$." Let $X_{1}, \cdots$, $X_{n-1} \in F_{\sigma}$ be mutually orthogonal. Apply (3) to these vector fields and notice that, because of the assumed orientations of $\dot{\sigma}(a)$ and $\dot{\sigma}(b)$, the terms representing mean curvatures make a contribution $\leqq 0$. By the hypothesis on Ricci curvature, the integral is also $<0$, so $\sum I_{\sigma}\left(X_{m}\right)<0$. It follows that $I_{\sigma}\left(X_{m}\right)<0$ for some $m$, so the $N$ variation $V(t, \epsilon)=\exp _{\sigma(t)}\left[\epsilon X_{m}(t)\right]$ is length-decreasing. Therefore, $\sigma$ could not be minimal.

Now, suppose $\alpha \in \pi_{1}(M, \bar{U})$ and let $c \in \alpha$. It is easily seen that $c$ is $\bar{U}$-homotopic to a path-product $\sigma_{1} \theta_{1} \sigma_{2} \theta_{2} \cdots \theta_{k-1} \sigma_{k}$, where each $\sigma_{i}:\left[a_{i}, b_{i}\right] \rightarrow M$ is a minimal $N$-geodesic intersecting $N$ only at its endpoints, while $\theta_{i}:\left[b_{i}, a_{i+1}\right] \rightarrow M$ lies in $N$. By the argument given above, it is impossible that $\dot{\sigma}_{i}\left(a_{i}\right)$ points "out of $U$ " while $\dot{\sigma}_{i}\left(b_{i}\right)$ points "into $U$," since $\sigma_{i}$ is to be minimal. Therefore, $c$ lies in $\bar{U}, \alpha$ is trivial and so $\pi_{1}(M, \bar{U})=\pi_{1}(M, U)$ is trivial, completing the proof.

As particular cases of the hypotheses of Theorem 1, we can require $N$ to be minimal (mean curvature $\equiv 0$ ) or, more stringently, totally geodesic. A sufficient condition for $N$ to have semidefinite mean curvatures is that the second fundamental form, itself, be semidefinite. Included here is the case where $N$ is totally umbilical; that is, $S_{v}$ is a fixed multiple of the metric tensor when $\nu$ of $N$ varies within a given orientation.

Notice that, if $N$ is minimal, the deformations in the proof can be used to retract all of $c$ into $N$, so that we recover the principal theorem in Frankel [3].

Let $M$ be a compact 3-manifold with positive Ricci curvatures and suppose $\pi_{1}(M)$ cannot be generated by less than $r$ elements. Suppose 
$M=T_{1} \cup T_{2}, N=T_{1} \cap T_{2}$, is a decomposition of $M$ into two solid tori $T_{1}$ and $T_{2}$, each bounded by the smooth surface $N$ of genus $g$. If $T_{1}$, say, is a homeomorph of a "standard" solid torus, then $\pi_{1}\left(T_{1}\right)$ is a free group on $g$ generators. Applying Theorem 1, we get

Corollary 1. Let $M$ be a compact 3-manifold with positive Ricci curvatures whose fundamental group cannot be generated by less than $r$ elements and let $T$ be a standard solid torus of genus $g$. If $r>g$, there is no smooth embedding of $T$ into $M$ such that the boundary of $T$ becomes a surface of semidefinite mean curvatures and the interior of $T$ goes onto the exterior of that surface.

II. Let $M$ be complete and Riemannian and let $N$ be a compact smooth hypersurface in $M$. The notations will be as in $\S$ I. If $U(\delta)$ $=\left\{v \in N^{\perp} \mid\|v\|<\delta\right\}$, define the thickness of $N$ to be

$$
\tau_{N}=\sup \{\delta \mid \exp \text { is one-to-one on } U(\delta)\} .
$$

It is easy to see that either $N$ has a focal point at distance $\tau_{N}$ or there are distinct, $v, w \in N^{\perp}\left(\|v\|=\|w\|=\tau_{N}\right)$ such that $\exp v=\exp w$ (cf. [10]).

M. Berger [1] has given an extension of Rauch's Comparison Theorem (cf. [7]) which shows that, if $M$ has positive sectional curvatures bounded above by 1 while $N$ is totally geodesic, no focal points of $N$ can be at distance less than $\pi / 2$. We will show an even stronger result: $\tau_{N} \geqq \pi / 2$.

We need the following lemma, true without curvature restrictions on $M$ and $N$ (cf. [4]).

LEMma. Let $v, w \in \mathfrak{T}^{\perp}, v \neq w$, with $\exp \tau_{N} v=\exp \tau_{N} w$. Let $b, c:\left[0, \tau_{N}\right]$ $\rightarrow M$ be geodesics parametrized by arc-length with $\dot{b}(0)=v, \dot{c}(0)=w$. Suppose the parameter-value $\tau_{N}$ is not focal for either $b$ or $c$. Then $\Varangle\left(\dot{b}\left(\tau_{N}\right), \dot{c}\left(\tau_{N}\right)\right)=\pi$.

Proof. Let $q=\exp \tau_{N} v=\exp \tau_{N} w$. Sufficiently small neighborhoods of $v$ and $w$ in the closed hypersurface $\partial U\left(\tau_{N}\right) \subset N^{\perp}$ are mapped by $\exp *$ into sets $V$ and $W$ intersecting at $q$. Since $\tau_{N}$ is not focal for $b$ or $c, V$ and $W$ are hypersurfaces near $q$ and, by Gauss' Lemma, are orthogonal, respectively, to $b$ and $c$. If $\Varangle\left(\dot{b}\left(\tau_{N}\right), \dot{c}\left(\tau_{N}\right)\right) \neq \pi, V$ and $W$ meet transversely at $q$, and it is easy to see that one can then find distinct $v_{1}, w_{1} \in N^{\perp},\left\|v_{1}\right\|=\left\|w_{1}\right\|<\tau_{N}$, with $\exp v_{1}=\exp w_{1}$. This contradicts the definition of $\tau_{N}$.

THEOREM 2. Let $M$ have positive curvatures bounded above by 1 and let $N$ be a compact totally geodesic hypersurface. Then $\tau_{N} \geqq \pi / 2$.

Proof. Suppose $\tau_{N}<\pi / 2$. Then no vector in $U\left(\tau_{N}\right)$ is focal. By the 
remarks above, there must be two geodesics, $b, c:\left[0, \tau_{N}\right] \rightarrow M$, parametrized by arc-length, such that $\dot{b}(0), \dot{c}(0) \in \Re^{\perp}$ and $b\left(\tau_{N}\right)$ $=c\left(\tau_{N}\right)$. Since $\tau_{N}<\pi / 2$, Berger's Comparison Theorem and the Lemma show that $\Varangle\left(\dot{b}\left(\tau_{N}\right), \dot{c}\left(\tau_{N}\right)\right)=\pi$. Therefore, $b$ and $c$ fit together into an $N$-geodesic $\sigma$ given by

$$
\begin{aligned}
\sigma(t) & =b(t), & 0 & \leqq t \leqq \tau_{N}, \\
& =c\left(2 \tau_{N}-t\right), & \tau_{N} & \leqq t \leqq 2 \tau_{N} .
\end{aligned}
$$

Let $X \in \mathcal{F}_{\sigma}$. Since $N$ is totally geodesic, $S_{\nu} \equiv 0$ for all $\nu \in \mathfrak{T}^{\perp}$. Therefore, by formula (2),

$$
I_{\sigma}(X)=-\int_{0}^{2 \tau_{N}} K(X(t)) d t<0 .
$$

Define an $N$-variation $V:\left[0,2 \tau_{N}\right] \times J \rightarrow M$ by $V(t, \epsilon)=\exp _{\sigma(t)}[\epsilon X(t)]$. Then $V$ is length-decreasing for small enough $J: \mathfrak{L}\left(V_{\epsilon}\right)<\mathfrak{L}\left(V_{0}\right)=\mathscr{L}(\sigma)$ for $\epsilon \in J-\{0\}$. In particular, the distance from $V_{\epsilon}(t)$ to $N$ is $<\tau_{N}$ for all $t$ and all $\epsilon \neq 0$. Therefore, by definition of $\tau_{N}$, there is a lifting of $V$ to $N^{\perp}, \Phi:\left[0,2 \tau_{N}\right] \times J \rightarrow N^{\perp}$, such that $\exp \circ \Phi=V$. Let $\{\epsilon(k)\}$ be a null sequence. Since $M$ is complete, $\exp U\left(\tau_{N}\right)$ has compact closure and, since $M$ and exp are smooth, it is easy to see that the set of lengths $\left\{\mathscr{L}\left(\Phi_{\epsilon}\right)\right\}$ is bounded. Thus, it may be assumed that there is a $\Phi_{0}:\left[0,2 \tau_{N}\right] \rightarrow N^{\perp}$ such that image $\Phi_{0} \subset \mathrm{Cl} U\left(\tau_{N}\right), \Phi_{\epsilon(k)} \rightarrow \Phi_{0}$ pointwise, and (by shrinking $J$, if necessary) $\Phi_{0}(0) \neq \Phi_{0}\left(2 \tau_{N}\right)$. By continuity, $\exp \circ \Phi_{\epsilon(k)} \rightarrow \exp \circ \Phi_{0}$, while $\exp \circ \Phi_{\epsilon(k)} \rightarrow \sigma$ by the construction of $V$. Thus, exp $\circ \Phi_{0}=\sigma$. But, geodesic rays in $M$ normal to $N$ must lift to rays in the fibers of $N^{\perp}$. Since fibers of $N^{\perp}$ are mutually disjoint, we get a contradiction. Therefore, $\tau_{N} \geqq \pi / 2$, as was to be shown.

It might be conjectured that if, instead of $N$ being totally geodesic, it were merely required that $N$ have small normal curvatures, the conclusion of Theorem 2 would hold with $\pi / 2$ replaced by a smaller lower bound for $\tau_{N}$ depending upon the normal curvatures of $N$, with this lower bound tending to $\pi / 2$ as the normal curvatures tend to 0 . This conjecture is false, as is shown by the following example, pointed out by Frankel.

Let $M$ be a real projective $n$-space, supplied with the Riemannian metric of constant curvature 1 . Let $N$ be an $(n-1)$-dimensional projective hyperplane. Then, $N$ is totally geodesic. Approximate $N$ by the embedded sphere $S(\alpha)=\left\{\exp \alpha v \mid v \in \Re^{\perp}\right\}$, where $\alpha$ is small. Then, the normal curvatures of $S_{\alpha}$ tend to 0 as $\alpha \rightarrow 0$. But $\tau_{S(\alpha)}=2 \alpha$, which tends toward 0 and not $\pi / 2$.

In this example, we notice that $S_{\alpha}$ bounds a disc $D_{\alpha}$ and the pair $\left(D_{\alpha}, S_{\alpha}\right)$ is a manifold-with-boundary embedded in $n$-dimensional 
projective space. For topological reasons, $S_{\alpha}$ has a collar neighborhood in $D_{\alpha}$; that is, $S_{\alpha}$ has a neighborhood in $D_{\alpha}$ homeomorphic to $S_{\alpha} \times[0,1)$. In this case, we can take the fibers of the collar neighborhood to be geodesics normal to $S_{\alpha}$ and of any length less than $(\pi / 2)-\alpha$. We can ask for a general estimate of the thickness of such a geodesic collar neighborhood.

Theorem 3. Let the manifold-with-boundary pair $(L, N)$ be embedded in $M$ so that $N$ is a smooth hypersurface separating $M$. Let the second fundamental form $S_{\nu}$ satisfy $S_{\nu}(X, X) \geqq \Delta\|X\|^{2}(\Delta \geqq 0)$ for the orientation of $\mathfrak{\Upsilon}^{\perp}$ corresponding to L. Finally, let $M$ have positive sectional curvatures bounded above by 1 . Then $N$ has a geodesic collar neighborhood in $L$ of thickness at least $\cot ^{-1} \Delta$.

Proof. Let $\Theta \subset \Re^{\perp}$ be the orientation of $\Re^{\perp}$ corresponding to $L$. Let $\Theta(\delta)=\{u \nu \mid 0 \leqq u<\delta, \nu \in \Theta\}$, and let $\tau_{(L, N)}=\sup \{\delta \mid \exp$ is one-toone on $\Theta(\delta)\}$. According to a generalization of Berger's Comparison Theorem due to F. Warner [9], no geodesic normal to $N$ and entering $L$ can have a focal point for $N$ until arc-length at least $\cot ^{-1} \Delta$. Assume $\tau_{(L, N)}<\cot ^{-1} \Delta$. By the same method as is used in proving the Lemma and Theorem 2, there can be constructed an $N$-geodesic $\sigma:\left[0,2 \tau_{(L, N)}\right] \rightarrow M$, lying in $L$. Let $X \in \mathcal{F}_{\sigma}$ be parallel and unitary. Using (2),

$$
I_{\sigma}(X) \leqq-\Delta-\Delta-\int_{0}^{2 \tau(L, N)} K(X(t)) d t<0 .
$$

The proof is completed in the same manner as the proof of Theorem 2.

REMARKs. $\cot ^{-1} \Delta \rightarrow \pi / 2$ as $\Delta \rightarrow 0$, so Theorem 2 is the limiting case of Theorem 3. The estimate $\pi / 2$ is best possible for Theorem 2 as is shown by the unit $n$-sphere with a unit $(n-1)$-sphere embedded as a totally geodesic submanifold.

\section{REFERENCES}

1. M. Berger, An extension of Rauch's metric comparison theorem and some applications, Illinois J. Math. 6 (1962), 700-712.

2. T. Frankel, Manifolds with positive curvalure, Pacific J. Math. 11 (1961), 165174.

3. - On the fundamental group of a compact minimal submanifold, Annals of Math. 83 (1966), 68-73.

4. R. Hermann, Focal points of closed submanifolds of Riemannian spaces, Nederl. Akad. Wetensch. Proc. 66 (1963), 613-628.

5. W. Klingenberg, Riemannsche Geometrie im Grossen (Lecture notes prepared by D. Gromoll and W. Meyer), Bonn, 1962.

6. M. Morse, The calculus of variations in the large, Amer. Math. Soc. Colloq. Publ., Vol. 18, Amer. Math. Soc., Providence, R. I., 1934. 
7. H. Rauch, $A$ contribution to differential geometry in the large, Ann. of Math. 54 (1951), 38-55.

8. J. L. Synge, On the neighborhood of a geodesic in Riemannian space, Duke Math. J. 1 (1935), 527-537.

9. F. Warner, Extension of the Rauch comparison theorem to submanifolds, Trans. Amer. Math. Soc. 122 (1966), 341-356.

10. J. H. C. Whitehead, On the covering of a complete space by the geodesics through a point, Ann. of Math. 36 (1935), 679-704.

InSTITUTE FOR AdVANCEd STUdy AND

University of California, Los ANgeles 